\title{
Performance of cognitive radar detection under EMI environment
}

\author{
Yuan Rufang ${ }^{1,2}$, Gan Rongbing ${ }^{1}$, Tang Guangfu² \\ 1.Science and Technology on Electronic Information Control Laboratory, Chengdu, China \\ 2.China Academy of Telecommunications Technology, Beijing, China
}

Phone: +86-028-87550925, Email: bethyuan@163.com

\begin{abstract}
Keywords: cognitive radar, waveform, anti-interference, artificial intelligence
\end{abstract}
\begin{abstract}
Cognitive Radar can adjust its transmit waveform to get the best outperform in the alternative targets and environments with a close-loop feedback. It has been regarded as one of the tendency of radar in the future. The optimization waveform design method is one of the critical technologies of radar cognition. This article analyses the detection performance of the cognitive radar which uses the dynamic waveform optimization. Firstly, the signal model and waveform solved process are reviewed; secondly, the theory analysis of performance decrease under the jamming is derived. Finally, the MTLAB numerical simulation results of deferent jamming are given.
\end{abstract}

\section{Introduction}

There is a sharp increase of equipments of two sides in the modern battlefield, such as radars, communication device and navigation device. The electromagnetic signals radiation of these equipments as well as the commercial communication signals make the electromagnetic environment quite congest. It's a big challenge for radar to precisely complete detection, identification, and tracking task faced with these irrelevant interference. Traditional radar uses a fixed transmission waveform, because radar's range and velocity resolution are closely related with the signal waveform, and when the radar target is not a point target or under clutter, the output signal to noise ratio(SNR) of the matched filter is affected by the power spectrum density(PSD) of transmission waveform[1]. And the SNR further affect the detection range of radar. Under such variable and complicated condition, the only adaptive data processing in receiver cannot achieve the satisfying performance. At the same time, the ultra large scale integrated circuit has laid the foundation for the high speed signal processing, the development of modern digital technology also gave birth to the direct waveform synthesis technology. All kinds of good hardware conditions make the adaptive processing of the transmitter become possible. Under this background, the concept of cognitive radar is proposed to provide a direction for the development of modern radar. Cognitive radar[2] could adjust the transmitter parameters to achieve a full match with the external objectives and environment, according to historical experience and the corresponding results of learning, reasoning, planning current echo, so as to optimize the performance of the work. Therefore, since proposed, cognitive radar has received a wide range of attention.

Cognitive radar system is shown in Figure 1, Compared with conventional adaptive radar, cognitive radar provides a physical feedback loop from the receiver to the transmitter, which can lead the transmitter to adjust the emission waveform. At the receiver, according to the environmental information (such as temperature, humidity, pressure, etc.) and prior knowledge (such as terrain, non cooperative target characteristics, etc.), the Bias tracker continues to monitor 
the target, establish the model of target and clutter via analyzing data, then feed it back to the transmitter. According to the feedback information, the emission waveform is optimized to meet the operational requirements according to certain criteria. Cognitive radar is realized by the online real time closed-loop feedback. It can realize the automatic "cognition" process of radar, so that it can be flexibly used in different working conditions (such as detection, recognition, tracking, etc.) and different environment.

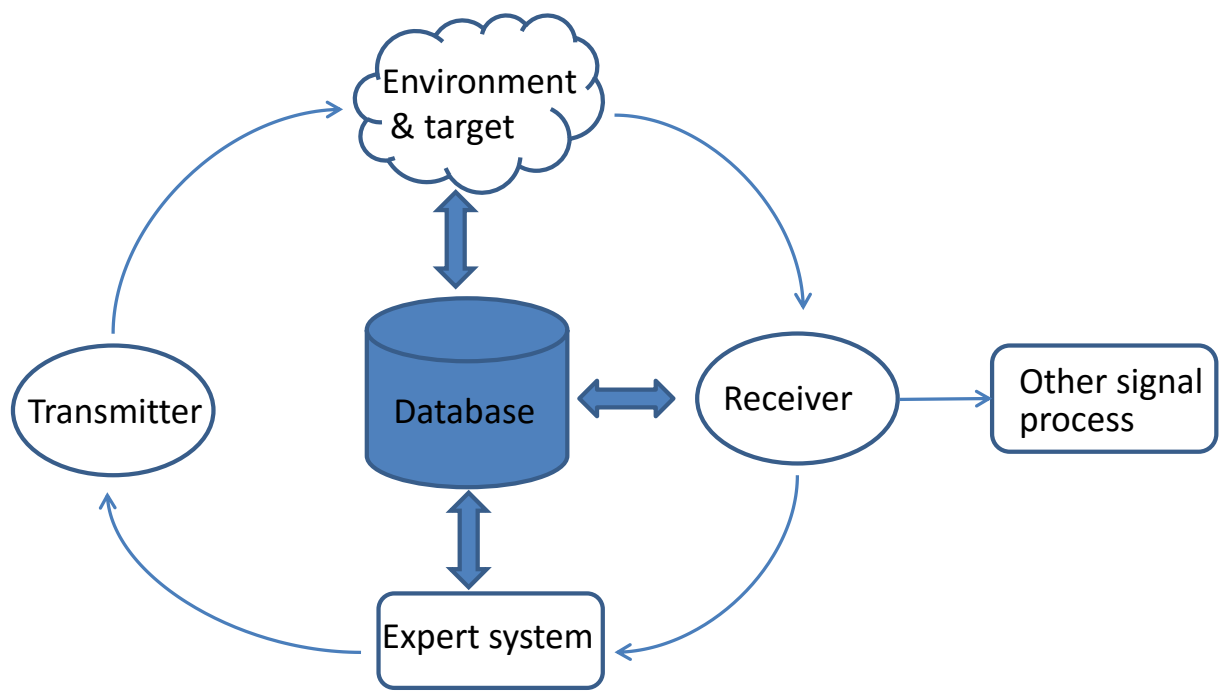

Fig 1 diagram of cognitive radar system

The proposal of CR direct the development of radar, while waveform design and self-adaption is the critical technology of $\mathrm{CR}$, as well as the hot zone of radar research[3]. Waveform design is the process of deciding the waveform parameters according to some norm under certain limitation (usually transmit power limitation). The main norms including SNR norm and mutual information norms, although another norm called minimal free-energy was proposed in [4], it turn out the same result with mutual information. In the problem of target detection, the detection probability is positively associated with signal noise ratio (SNR) at the receiver under a constant false alarm probability, therefore, waveform optimized by SNR norms can improve the detection performance efficiently. Scholars have put forward kinds of optimize method under SNR norm: article [5] discussed the problem in frequency domain and solved the power spectral density of optimal waveform, Joseph R. Guerci stated several optimize methods in the bases of solving eigenmatrix in [6], including the case of colored noise background and signal depend clutter background. Then [7] carried out an improvement under a certain background in terms of the method in [6]. All of articles above emphasize on analysis of optimize method and effect but scarcely discuss decrease of the performance while facing with strong jamming. However there are always interference and hostile jam. Research on anti-interference performance of cognitive radar waveform can help to make protection for the weak segment at the beginning of the design. Also for countermeasure, it offers the theoretical basis of jam and anti-jam strategy to cognitive radar in the future.

This article introduces the most widely discussed optimal method of cognitive radar waveform, then it analyzes the effects that stable and unstable jamming caused to the pulse accumulation and detection performance, and finally the quantitative simulation results are displayed and explained.

\section{Signal Model}

With colored jamming, the cognitive radar detection system model is depicted in Fig $2^{[6]}$. 


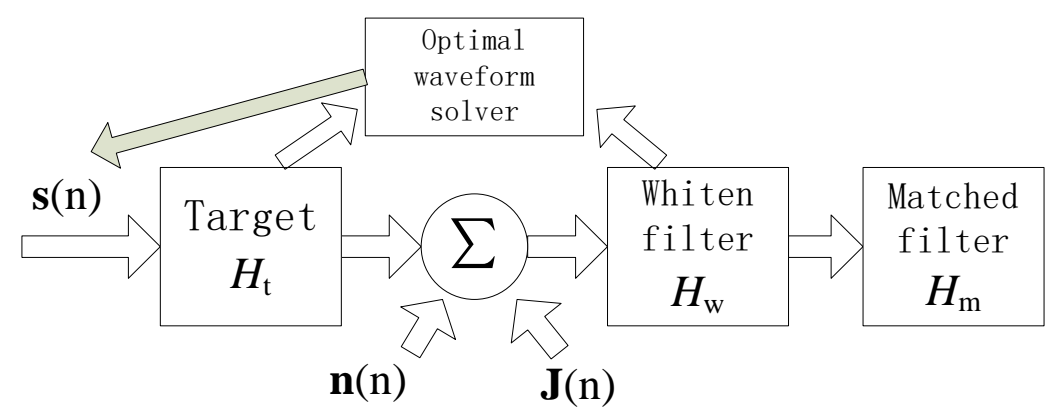

Fig 2 diagram of cognitive radar detection system

Radar signal $\mathbf{s}(\mathrm{n})$ meets target and scatter backward, with additive system noise $\mathbf{n}(\mathrm{n})$ (colored) and hostile jamming $\mathbf{J}(\mathrm{n})$, are put in whiten filter, then filtered by matched filter and input detector comparing with threshold. The input vector of whiten filter is

$$
\mathbf{x}(n)=\mathbf{H}_{t} \mathbf{s}(n)+\mathbf{J}(n)+\mathbf{n}(n)
$$

where $\mathbf{H}_{t}$ is target response matrix, and possesses the following Toeplitz form, here $\mathrm{h}_{i}$ is target pulse response, $\quad \mathrm{N}_{h}$ is the length of $\mathrm{h}_{i}, \mathrm{~N}_{s}$ is the length of transmit signal sequence.

$$
\mathbf{H}_{t}=\left[\begin{array}{cccc}
h_{0} & 0 & \cdots & 0 \\
h_{1} & h_{0} & \cdots & 0 \\
\vdots & \vdots & \vdots & \vdots \\
h_{N_{h}-1} & h_{N_{h}-2} & \ddots & \vdots \\
0 & h_{N_{h}-1} & \ddots & \vdots \\
\vdots & \vdots & \ddots & \vdots \\
\vdots & \vdots & \ddots & \vdots
\end{array}\right]_{\left(N_{h}+N_{s}-1\right) \times N_{s}}
$$

Assuming that the interference and noise are unrelated, from Wiener filtering theory [8], the response of the whiten filter is $\mathbf{H}_{t}=\mathbf{R}_{j n}{ }^{-1 / 2}$, where

$$
\mathbf{R}_{j n}=E\left\lfloor(\mathbf{J}(n)+\mathbf{n}(n))(\mathbf{J}(n)+\mathbf{n}(n))^{T}\right\rfloor
$$

is the autocorrelation of combination of the input colored noise and jamming, it's a $\mathrm{N}$ dimension positive define Hermitian matrix. Where $\mathrm{N}=\mathrm{N}_{h}+\mathrm{N}_{s^{-}}-$.

The response of the matched filter is

$$
\mathbf{H}_{m}=\mathbf{x}_{s}(n)=\mathbf{H}_{w} \mathbf{H}_{t} \mathbf{s}(n)
$$

and the output is

$$
\begin{aligned}
\mathbf{y}(n) & =\mathbf{H}_{m} \mathbf{H}_{w} \mathbf{x}(n) \\
& =\mathbf{H}_{m} \mathbf{H}_{w} \mathbf{H}_{t} \mathbf{s}(n)+\mathbf{H}_{m} \mathbf{H}_{w} \mathbf{J}(n)+\mathbf{H}_{m} \mathbf{H}_{w} \mathbf{n}(n) \\
& =\mathbf{y}_{s}(n)+\mathbf{y}_{j}(n)+\mathbf{y}_{n}(n)
\end{aligned}
$$


Where

$$
\begin{aligned}
& \mathbf{y}_{s}(n)=\mathbf{H}_{m} \mathbf{H}_{w} \mathbf{H}_{t} \mathbf{s}(n) \\
& \mathbf{y}_{j}(n)=\mathbf{H}_{m} \mathbf{H}_{w} \mathbf{J}(n) \\
& \mathbf{y}_{n}(n)=\mathbf{H}_{m} \mathbf{H}_{w} \mathbf{n}(n)
\end{aligned}
$$

respectively represent the signal part, jamming part, and noise part of the output. For convenience, when jamming exists, calculate it together with noise. Therefore, after two filters, the output SNR is

$$
S N R=\frac{\left|\mathbf{y}_{s}(n)\right|^{2}}{E\left\{\left|\mathbf{y}_{j}(n)+\mathbf{y}_{n}(n)\right|^{2}\right\}}=\left|\mathbf{s}^{H}(n) \mathbf{H}_{t}^{H} \mathbf{R}_{j n}^{-1} \mathbf{H}_{t} \mathbf{s}(n)\right|
$$

In practical application, the power of the transmitted waveform is limited by the transmitter. And the SNR criterion is to choose the optimal waveform to make the SNR maximal. So it can be described as the following optimization problem, where $\mathrm{P}$ is the maximum output power of the transmitter.

$$
\left\{\begin{array}{l}
\max _{\{\mathbf{s}(n)\}}\left|\mathbf{s}^{H}(n) \mathbf{H}_{t}^{H} \mathbf{R}_{j n}^{-1} \mathbf{H}_{t} \mathbf{s}(n)\right| \\
\text { s.t } \mathbf{s}^{H}(n) \mathbf{s}(n) \leq P
\end{array}\right.
$$

Let $\mathbf{Q}=\mathbf{H}_{t}{ }^{\mathrm{H}} \mathbf{R}_{j n}{ }^{-1} \mathbf{H}_{t}$ and name it as object matrix, the solution of problem (10) is

$$
\mathbf{s}_{\text {opt }}=\sqrt{P} \mathbf{u}_{\max } /\left\|\mathbf{u}_{\max }\right\|
$$

where, $\lambda_{\max }$ is the maximum eigenvalue of $\mathrm{Q}$, and $\mathbf{u}_{\max }$ is responsible eigenvector.

\section{Performance of Cognitive Radar with Jamming}

The ideal jamming conditions that optimal signal in (11) applicable are: a) The interference signal is the generalized stationary or in a certain time can be regarded as the generalized stationary, and it is ergodic; b) Interference signal and transmit signal is not relevant; c) The interference signal spectrum is not uniform in the radar signal frequency band.

As $J$ is stationary random process, its statistical properties will not change with time, and $J$ is ergodic, we can substitute set average with time average to calculate the two-moment characteristics of $J$. Therefore, the correlation function is

$$
r_{j}(\tau)=\lim _{T \rightarrow \infty} \frac{1}{T} \int_{0}^{T} j(t+\tau) j(t) d \tau
$$

The discrete form is

$$
r_{j}(k)=\lim _{N \rightarrow \infty} \frac{1}{N} \sum_{n=0}^{N-1} j(n+k) j(n)
$$

In practical engineering applications, the infinite signal is not possible, and because of the limitation of radar receiver processing data length and real-time response, the finite $M$ point is 
generally used as a sample to estimate the correlation function. That is

$$
\tilde{r}_{j}(k)=\frac{1}{M} \sum_{n=0}^{M-1} j(n+k) j(n)
$$

According to the deduction above and the conjugate symmetry of correlation function, the estimated value of $\mathbf{R}$ is

$$
\tilde{R}=\left[\begin{array}{llll}
\tilde{r}(0) & \tilde{r}(1) & \cdots & \tilde{r}(M-1) \\
\tilde{r}^{*}(1) & \tilde{r}(0) & \cdots & \tilde{r}(M-2) \\
\vdots & \vdots & \ddots & \vdots \\
\tilde{r}^{*}(M-1) & \tilde{r}^{*}(M-2) & \cdots & \tilde{r}(0)
\end{array}\right]
$$

Combined with the analysis of the previous section, when the interference signal is stationary and ergodic, the estimation of object matrix $\widetilde{\mathbf{Q}}=\hat{\mathbf{H}}_{t}^{H} \widetilde{\mathbf{R}}_{j n}^{-1} \hat{\mathbf{H}}_{t}$ is constant, then the optimal waveform $\mathbf{s}_{\text {opt }}=\sqrt{P} \mathbf{u}_{\text {max }} /\left\|\mathbf{u}_{\max }\right\|$ is constant too within a certain time, this is necessary for pulse accumulation and target Doppler detection in radar data process. However, if the cognitive radar are exposed to non-stationary jamming, the performance will decrease, and the degree of reduction is depend on the jamming itself. Next part shows the detail simulation.

\section{Simulation Results}

Consider a target $1 \mathrm{~km}$ far from radar, $\mathrm{RCS}=1 \mathrm{~m}^{2}$, the Doppler frequency is $100 \mathrm{~Hz}$; radar $\mathrm{PRF}=2 \mathrm{kHz}$, coherent pulse accumulation number is $\mathrm{M}=20$, the stationary jamming is

$$
J_{1}=\cos \left(2 \pi f_{1} t\right)+\cos \left(2 \pi f_{2} t\right)
$$

$f_{1}=20 \mathrm{MHz}$ and $f_{2}=40 \mathrm{MHz}$. The result of the conventional frequency line module (LFM) transmit signal $(\mathrm{B}=40 \mathrm{MHz}, \tau=25 \mathrm{us})$ in the same case is displayed to compare. Non-stationary jamming is

$$
J_{2}=\cos \left(\pi B_{1} \tau_{1} t^{2}\right)
$$

Case 1: stationary jamming When adding jamming $J_{1}, J S R=13 \mathrm{~dB}$ and $\mathrm{JSR}=19 \mathrm{~dB}$, the coherent accumulation result of two transmit signal is respectively shown in Fig 3 and Fig5. And the slice of range and doppler dimension at the peak is respectively shown in Fig 4 and Fig 6. Apparently, both of two waveform could detect the target range and doppler. In doppler dimension, the side-lobe of LFM is much higher than that of opt, while in range dimension, the opt seems not good as LFM, because of the 0.4 high false target. But consider the latter CFAR process, the two performance seemly almost same. But when the JSR rise to $19 \mathrm{~dB}$, both of the doppler and target range result of LFM is wrong, while the opt waveform could accurately measure the target range and velocity, nearly without performance decrease. It shows that the optimal waveform possesses far better anti-interference performance than traditional LFM wave. 

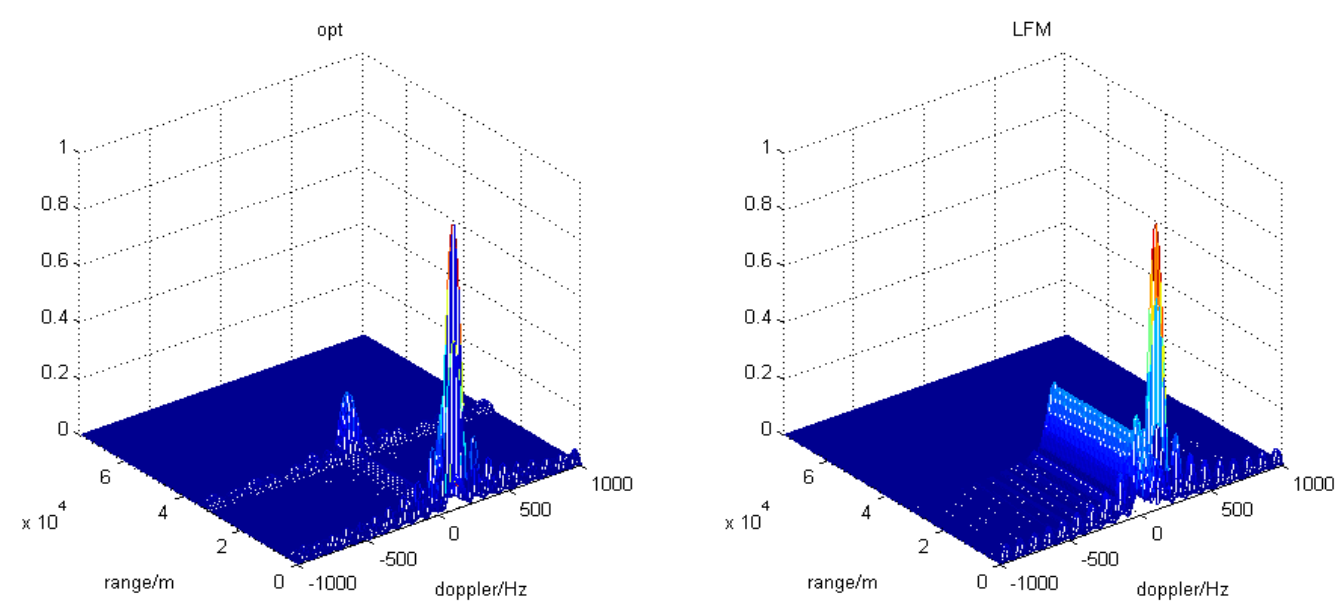

Fig 3 accumulation results of two waveform $(\mathrm{JSR}=13 \mathrm{~dB})$
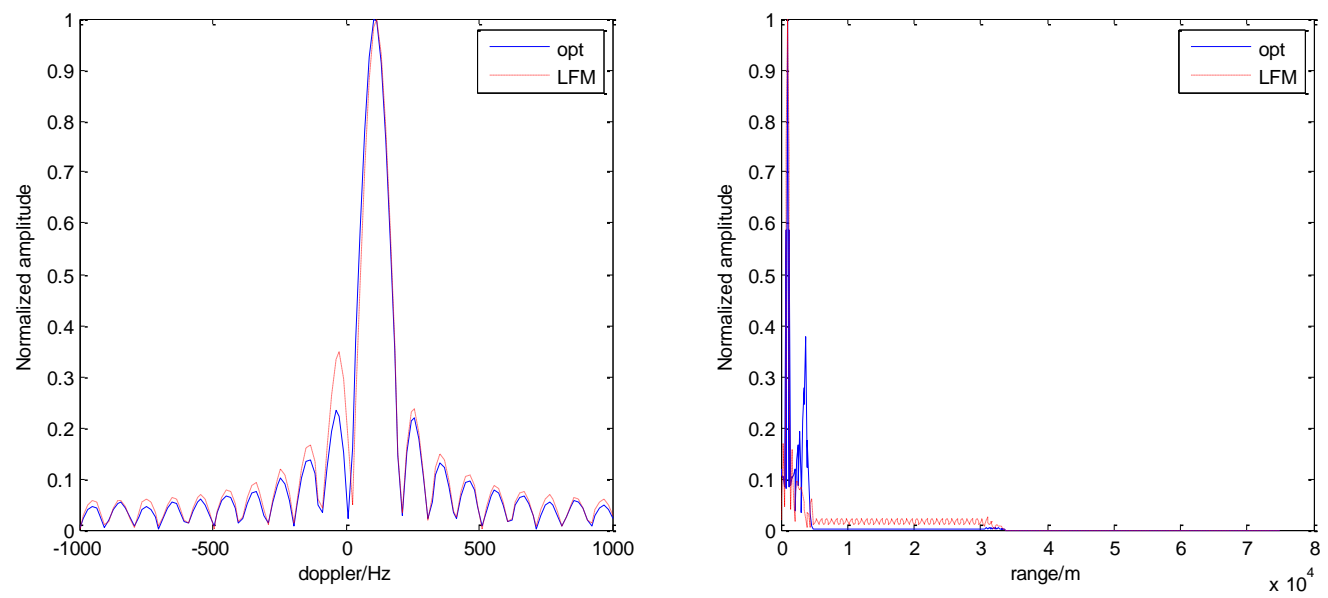

Fig 4 range and doppler slice $(\mathrm{JSR}=13 \mathrm{~dB})$
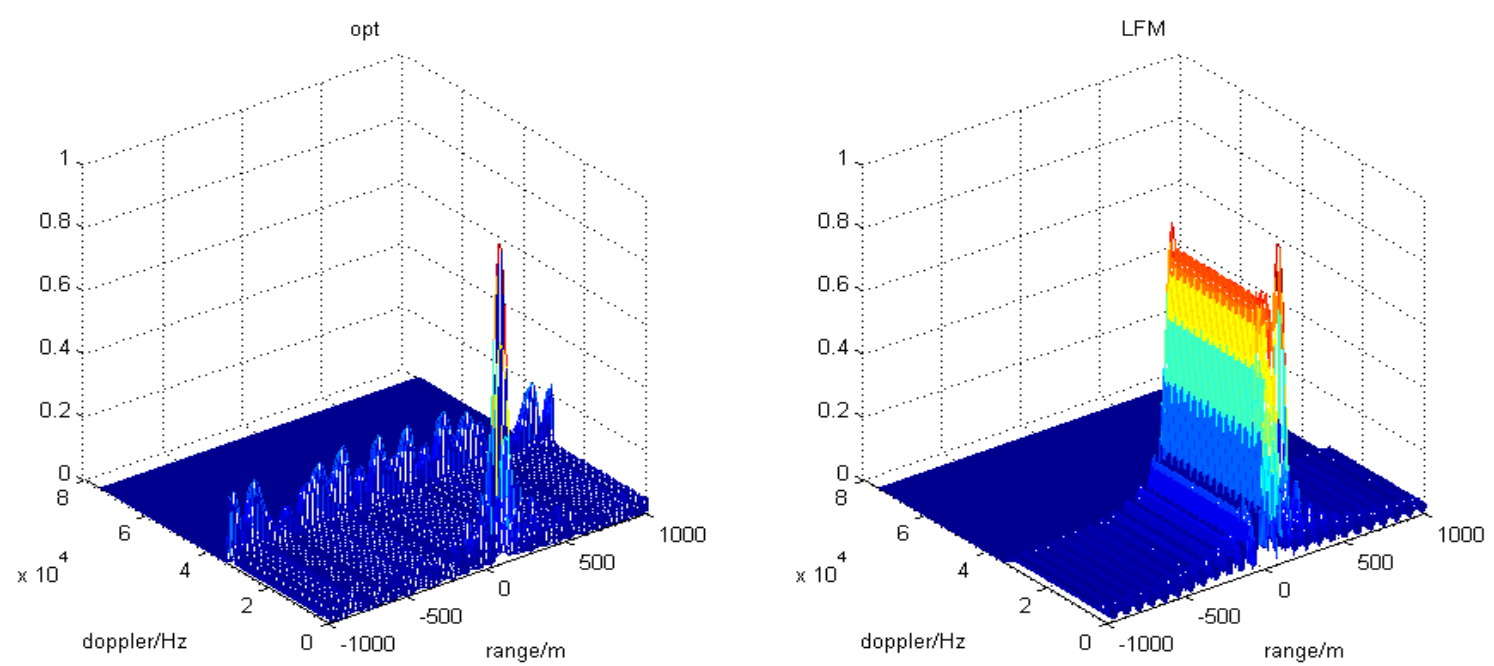

Fig 5 accumulation results of two waveform(JSR $=19 \mathrm{~dB})$ 

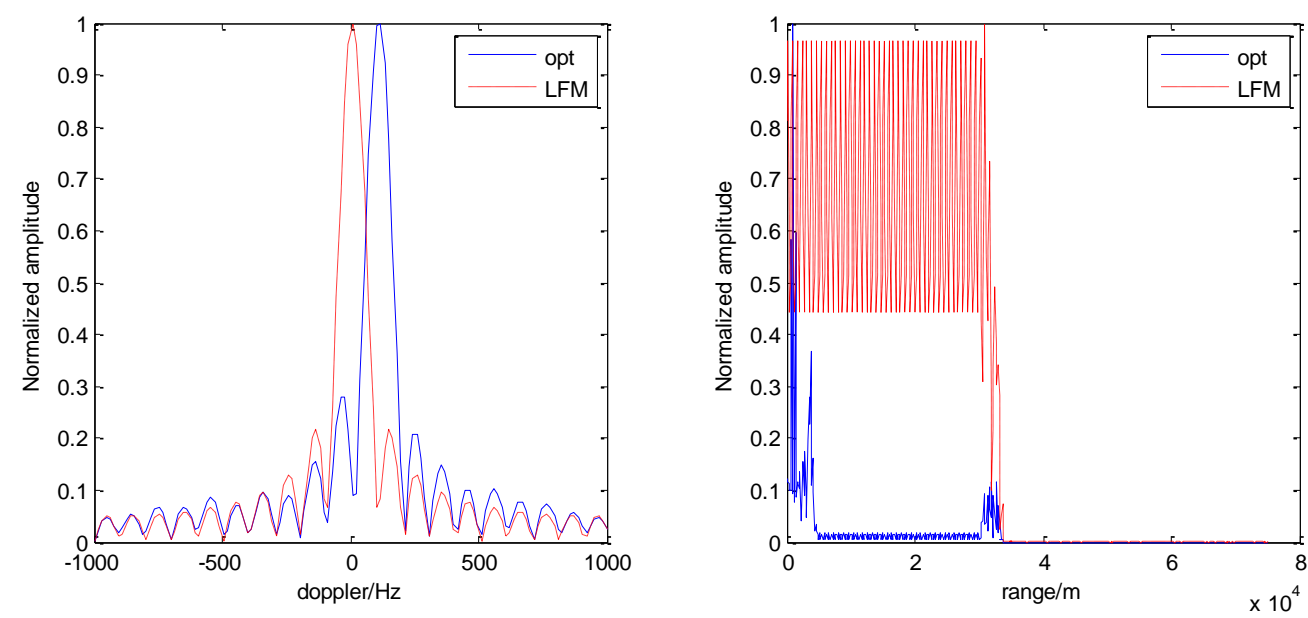

Fig 6 range and doppler slice (JSR=19dB)

Case 2: non-stationary jamming When adding jamming $J_{2}$, and $\mathrm{JSR}=13 \mathrm{~dB}$, the target range is $3 \mathrm{~km}$, and doppler frequency is $100 \mathrm{~Hz}$. The result of the accumulation of different parameters are displayed in Fig 7, these parameters are: (a) $\tau_{1}=0.5 \mathrm{~ms}, B_{1}=40 \mathrm{kHz} \quad$ (b) $\tau_{1}=0.5 \mathrm{~ms}, B_{1}=300 \mathrm{kHz}$ (c) $\tau_{1}=0.5 \mathrm{~ms}, B_{1}=500 \mathrm{kHz}$. In subfigure(a), the both of target range and doppler are accurately detected, in subfigure(b), target range are accurately detected while doppler is a little far from real value, in subfigure(c), both of target range and doppler can not be detected. Comparing the three subfigure in Fig 7, under the jamming with same power, as $B_{1}$ goes up, the performance of cognitive radar get worse and worse. That because with the constant $\tau_{1}, B_{1}$ represents the change speed of the jamming frequency, also the change speed of the statistical properties, and it brake the ideal interference condition a). The transmit signal is "chasing" the varying jamming, but when it changes too fast to "follow up", the performance of radar will decrease.
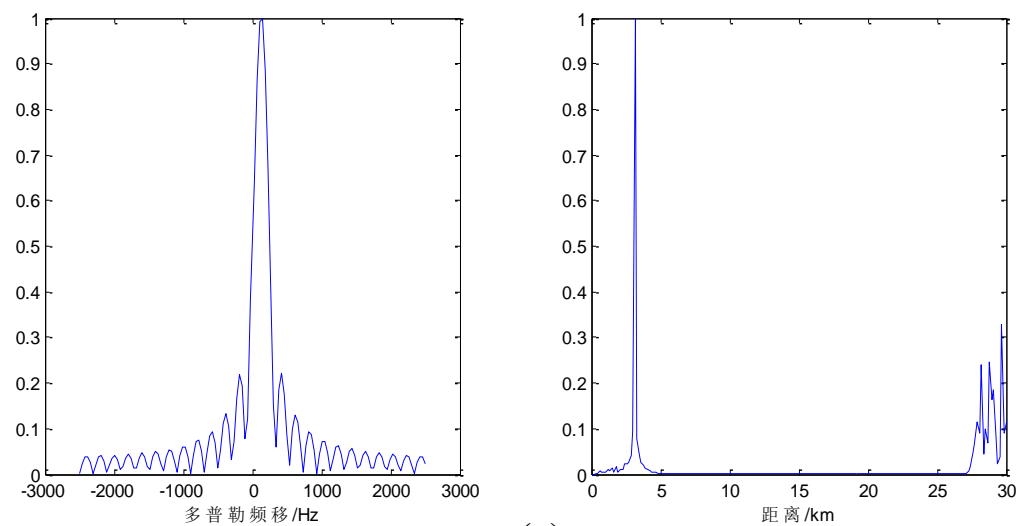

(a)
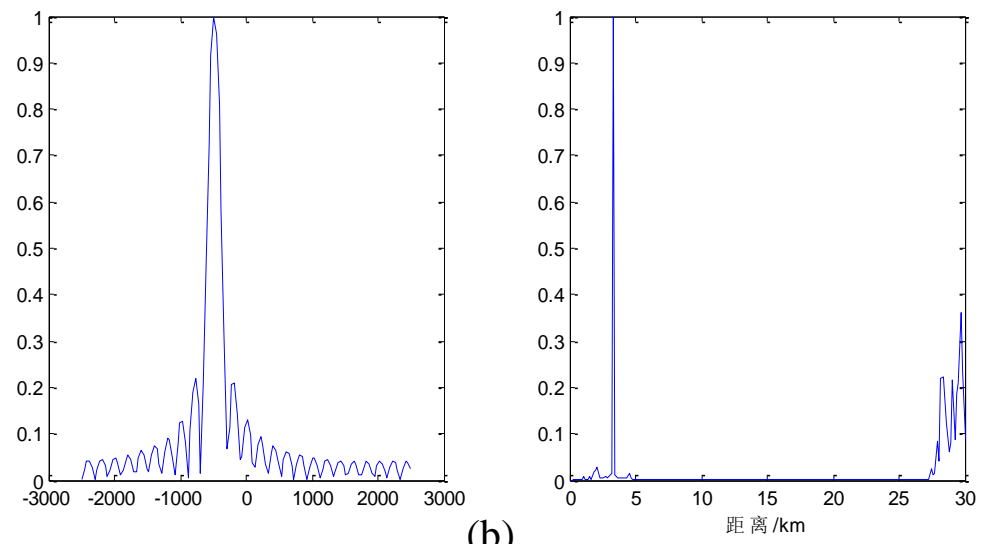

(b) 

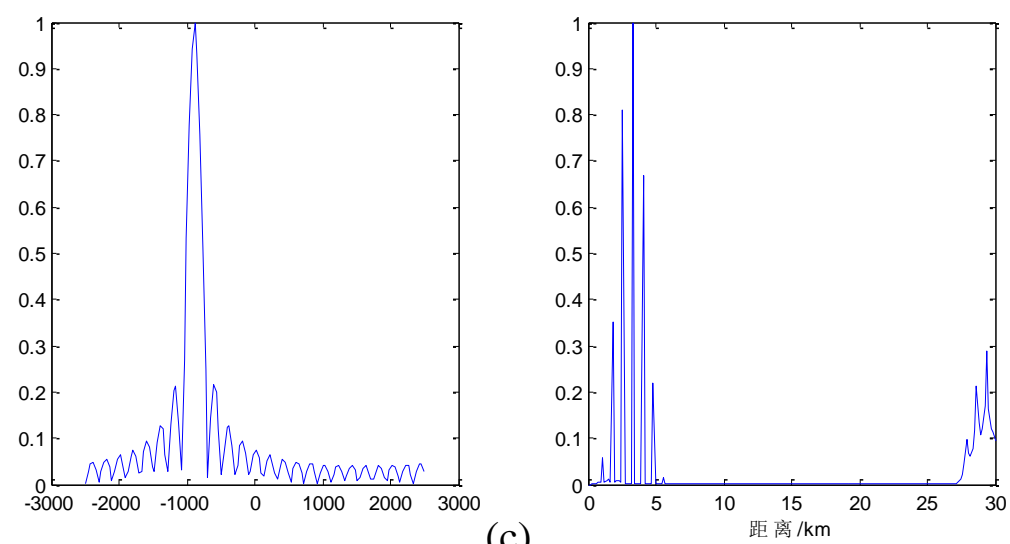

(c)

Fig 7 left: doppler slice/Hz; right: range slice/km

\section{Conclusion}

Automation and intelligence is the inevitable trend of the development of science and technology, and so it is in the radar field. Cognitive radar is the represent of the intelligent radar and shows better performance than traditional radar. The most important part of cognitive radar is the waveform design, and there are some kinds of criterion and method to solve the optimal waveforms of different radar application scene.

This article analysis the common waveform design method, when under weak jamming (JSR $<15 \mathrm{~dB}$ ), the optimal performs nearly the same with LFM, and when the jamming get stronger, the former performs far good than the latter. However, when the interference frequency is changing, the performance of optimal waveform will decrease.

\section{References}

[1] S.Haykin. Optimal waveform design for cognitive radar[J]. IEEE Asilomar 2008

[2] S.Haykin. Cognitive radar:a way of the future [J]. IEEE Signal Processing Magazine,2006,23(1):30-40

[3] Lixiang Fanmeimei. Research progress of cognitive radar and its key technologies[J]. Electronic journal 2012, 40(9):1683-1686

[4] Turlapaty,A; Yuanwei Jin, 'Parameter estimation and Waveform Design for Cognitive Radar by minimal free-energy principle,' Acoustics, Speech and Signal Processing, 2013 IEEE International Conference on, pp6244-6248

[5] Bin Wang; Jinkuan Wang; Xin Song; Yinghua Han, 'A new Waveform design Method for Cognitive Radar', Intelligent Information Technology Application, 2009 vol.2 p176-179

[6] Nijure,Y.; Yifan Chen; Boussakta,S.; Chau Yuen; Yong Huat Chew; Zhiguo Ding, 'Novel System Architecture and Waveform Design for Cognitive Radar Radio Networks,' Vehicular Technology,IEEE Transactions on, vol.61,no.8,pp.3630-3642,Oct.2012

[7] Guerci J R, Guerci R M, Ranagaswamy M, Bergin J S, Wicks M C. CoFAR: Cognitive fully adaptive radar[J] Radar Conference 2014, 19(23): 0984-0989.

[8] Harry L Van Trees. Detection, Estimation, and Modulation Theory[M]. Beijing, Electronics industry Press, 2007: 136-139. 